\title{
The Differential Association between Muscle Strength and Diabetes Mellitus According to the Presence or Absence of Obesity
}

\author{
Bo Kyung Koo* \\ Department of Internal Medicine, SMG-SNU Boramae Medical Center, Seoul National University College of Medicine, Seoul, Korea
}

Background: Muscle strength can be affected by body mass index. In the present study, we compared the association between the diabetes mellitus (DM) and muscle strength according to obesity.

Methods: We analyzed the association between DM and muscle strength using the Korea National Health and Nutrition Examination Survey 2014 to 2016 data weighted to represent the Korean population aged between 30 and 79 years old. Muscle strength was classified into age- and sex-specific quartiles (Qs) of handgrip strength, with the lowest Q defined as "low muscle strength (LMS)."

Results: Muscle strength was positively associated with body mass index in both sexes $(P<0.001)$; the prevalence of obesity increased by $30 \%$ in male (odds ratio [OR], 1.300; 95\% confidence interval [CI], 1.231-1.373) and $12 \%$ in female $(\mathrm{OR}, 1.122 ; 95 \% \mathrm{Cl}, 1.062-1.185)$, respectively, per one $\mathrm{Q}$ of muscle strength. In contrast, the prevalence of DM decreased as muscle strength increased (OR per one $\mathrm{Q}, 0.926 ; 95 \% \mathrm{Cl}, 0.862-0.996$ in male and OR per one $\mathrm{Q}, 0.917 ; 95 \% \mathrm{Cl}, 0.854-0.986$ in female). LMS was significantly associated with DM even following adjustment for age, sex, family history of DM, abdominal obesity, dyslipidemia, and hypertension (OR, 1.328; 95\% $\mathrm{Cl}, 1.133-1.558)$. Stratified analysis according to obesity status showed that it remained significant only in nonobese populations (OR, 1.513; $95 \% \mathrm{Cl}, 1.224-1.870$ in nonobese participants and $\mathrm{OR}, 1.124 ; 95 \% \mathrm{Cl}, 0.879-1.437$ in obese participants).

Conclusion: LMS was independently associated with DM in the Korean population aged between 30 and 79 years. However, obesity-stratified analysis revealed that it was significant only in the nonobese population.
Received February 8, 2019

Reviewed February 26, 2019

Accepted March 13, 2019

*Corresponding author

Bo Kyung Koo

https://orcid.org/0000-0002-6489-2656

Department of Internal Medicine, SMG-SNU Boramae Medical Center Seoul National University College of Medicine, 20 Boramae-ro 5-gil, Dongjak-gu, Seoul 07061, Korea Tel: +82-2-870-2225

Fax: +82-2-831-2826

E-mail: bokyungkoomd@gmail.com

Key words: Sarcopenia, Muscle strength, Diabetes mellitus, Obesity, Korea

\section{INTRODUCTION}

Sarcopenia increases the risk of not only diverse metabolic diseases including diabetes mellitus $(\mathrm{DM})^{1,2}$ but also cardiovascular diseases $^{3}$ and long-term mortality. ${ }^{4}$ In the last decade, it has been reported that muscle quality as well as muscle mass is important in metabolic health ${ }^{5-7}$, and the measurement of muscle strength is one easy way to evaluate muscle quality. ${ }^{8,9}$ Muscle strength is significantly associated with metabolic health ${ }^{6,7}$, low cardiovascular events $^{10,11}$, and low all-cause mortality. ${ }^{5,10}$ Furthermore, the Health,
Aging and Body Composition study, which was designed to determine the role of body composition changes in the risk of poor health outcomes, showed that muscle strength is more important than muscle mass for predicting mortality. ${ }^{5}$

Body mass index (BMI) has been reported to be associated with muscle strength in diverse populations: absolute strength is higher in obese versus nonobese individuals ${ }^{12-15}$, which implicates that the presence of obesity should be considered in the analysis of the association between muscle strength and metabolic disease. We investigated the relationship between muscle strength and the preva-

Copyright (C) 2019 Korean Society for the Study of Obesity

(a) This is an Open Access article distributed under the terms of the Creative Commons Attribution Non-Commercial License (http://creativecommons.org/licenses/by-nc/4.0/) which permits unrestricted non-commercial use, distribution, and reproduction in any medium, provided the original work is properly cited. 
lence of DM according to obesity status among participants in the Korea National Health and Nutrition Examination Survey (KNHANES) from 2014 to 2016. As aging is one of the most important determining factors for both of muscle strength and diabetes, we used age-specific cutoff values for the definition of low muscle strength (LMS).

\section{METHODS}

\section{Subjects}

The KNHANES is conducted on noninstitutionalized Korean civilians using a stratified multistage probability-based sampling design. ${ }^{16}$ To ensure the results best represent the entire Korean population, weights are assigned to each respondent. ${ }^{17}$ For this study, we selected the KNHANES 2014 to 2016 data which provide the handgrip strength of participants. Based on the numbers of participants in the 2014, 2015, and 2016 KNHANESs ( $\mathrm{n}=$ $7,550,8,150$, and 8,127 , respectively), weights for the combined dataset were recalculated. Subjects aged 30 to 79 years were selected and, after excluding subjects whose handgrip strength was unavailable, 13,149 participants were finally included in our study. The KNHANES 2014 was approved by the Institutional Review Board (IRB) of the Korean Centers for Disease Control (IRB No. 2013-12EXP-03-5C). Separately, for the 2015 and 2016 KNHANESs, IRB approval was not required under the Bioethics Act.

\section{Metabolic parameter measurement}

The precise methods for the measurement of clinical parameters were reported previously. ${ }^{18}$ Briefly, cases of DM were defined as subjects who were using antidiabetic medications including insulin at the time of the survey, had hemoglobin Alc values of $48 \mathrm{mmol} / \mathrm{mol}$ (6.5\%) or more, or had 8-hour fasting plasma glucose levels of 7.0 $\mathrm{mmol} / \mathrm{L}$ or more. Hypertriglyceridemia was defined as a fasting triglyceride level of $150 \mathrm{mg} / \mathrm{dL}$ or more, while low high-density lipoprotein (HDL) cholesterol was defined as that less than $40 \mathrm{mg} / \mathrm{dL}$ and less than $50 \mathrm{mg} / \mathrm{dL}$ in male and female, respectively, according to the National Cholesterol Education Program criteria. ${ }^{19}$ Additionally, abdominal obesity was defined as a waist circumference of $90 \mathrm{~cm}$ or more and $85 \mathrm{~cm}$ or more in male and female, respective$1 y^{20}$, and metabolic syndrome was defined based on the revised $\mathrm{Na}$ - tional Cholesterol Education Program Adult Treatment Panel III criteria. ${ }^{19}$ Hypertension was defined as a blood pressure of $140 / 90$ $\mathrm{mmHg}$ or more or the use of antihypertensive medication. BMI was classified based on the World Health Organization's Asia-Pacific criteria, with that of $25 \mathrm{~kg} / \mathrm{m}^{2}$ or greater indicating obesity.

\section{Measurement of handgrip strength}

Handgrip strength was measured three times in each individual both in the dominant and nondominant arms using a digital grip strength dynamometer (TKK 5401; Takei, Tokyo, Japan) which can measure force between 5.0 and $100.0 \mathrm{~kg}$. During the assessment, participants were instructed to stand upright with their feet placed hip-width apart, to look forward with the elbow fully extended, and to squeeze the grip continuously with full force for at least 3 seconds. The resting time between each measurement was at least 60 seconds. Muscle strength in each individual was defined as the average of handgrip strength of dominant hand $(\mathrm{kg})$ from three trials, and its quartiles (Qs) were calculated in each sex at every 10 years of age (i.e., 30-39, 40-49, 50-59, 60-69, and 70-79 years). Q1 and Q4 were the lowest and highest Qs of handgrip strength, respectively; Q1 was specifically defined as the subjects with LMS.

\section{Statistical analyses}

All data were analyzed using the SPSS version 18.0 (SPSS Inc., Chicago, IL, USA). The results are presented in the format of mean (standard error $[\mathrm{SE}]$ ) or prevalence (\% and SE). Sampling weights were used to account for complex sampling and applied to all regression analyses. Logistic regression analysis adjusted for age, obesity, and other risk factors was used to identify differences in the prevalence of diabetes with respect to muscle strength $Q$. Linear regression analysis was used to analyze the associations between continuous variables and muscle strength $Q$. The level of significance was set at $P<0.05$.

\section{RESULTS}

\section{Clinical characteristics according to muscle strength}

The mean age of the study population aged 30 to 79 years of the 2014 to 2016 KNHANESs, was 50.7 years (SE, 0.2 years), and 49.4\% (SE, $0.4 \%$ ) were male. The prevalences of DM and obesity 
Table 1. Handgrip strength $(\mathrm{kg})$ in each age group

\begin{tabular}{|c|c|c|c|c|c|c|}
\hline \multirow{2}{*}{ Age (yr) } & \multicolumn{3}{|c|}{ Male } & \multicolumn{3}{|c|}{ Female } \\
\hline & Dominant arm & Nondominant arm & $P^{*}$ & Dominant arm & Nondominant arm & $P^{*}$ \\
\hline 30-39 & $43.6 \pm 0.3$ & $41.6 \pm 0.2$ & $<0.001$ & $25.4 \pm 0.1$ & $24.1 \pm 0.1$ & $<0.001$ \\
\hline $40-49$ & $42.5 \pm 0.2$ & $40.8 \pm 0.2$ & $<0.001$ & $25.1 \pm 0.1$ & $23.8 \pm 0.1$ & $<0.001$ \\
\hline $50-59$ & $39.6 \pm 0.2$ & $38.2 \pm 0.2$ & $<0.001$ & $24.0 \pm 0.1$ & $22.7 \pm 0.1$ & $<0.001$ \\
\hline $60-69$ & $36.4 \pm 0.2$ & $35.1 \pm 0.2$ & $<0.001$ & $22.1 \pm 0.1$ & $20.8 \pm 0.1$ & $<0.001$ \\
\hline 70-79 & $30.9 \pm 0.3$ & $30.1 \pm 0.2$ & $<0.001$ & $18.7 \pm 0.2$ & $17.8 \pm 0.2$ & $<0.001$ \\
\hline Pfor trend ${ }^{\dagger}$ & $<0.001$ & $<0.001$ & & $<0.001$ & $<0.001$ & \\
\hline
\end{tabular}

Values are presented as mean \pm standard error.

*Wilcoxon signed-rank test to compare handgrip strength between dominant arm and nondominant arm; †From logistic regression for complex sample.

were $13.5 \%$ (SE, $0.4 \%)$ and $36.0 \%$ (SE, $0.5 \%$ ), respectively. Mean handgrip strength of the dominant arm was $43.6 \mathrm{~kg}$ (SE, $0.3 \mathrm{~kg}$ ) and $25.4 \mathrm{~kg}$ (SE, $0.1 \mathrm{~kg}$ ) in male and female aged 30 to 39 years (Table 1); by contrast, that of male and female aged 70 to 79 years was $30.9 \mathrm{~kg}$ (SE, $0.3 \mathrm{~kg}$ ) and $18.7 \mathrm{~kg}$ (SE, $0.2 \mathrm{~kg}$ ), respectively. Overall, as age increased, the mean handgrip strength decreased significantly irrespectively of sex or dominant/nondominant arm (all $P<0.001)$ (Table 1). Considering the age-dependency of muscle strength, an age-adjusted handgrip strength parameter was thought appropriate for use in the further analyses. For that, Qs of handgrip strength were calculated for each sex encompassing ranges of 10 years of age (i.e., 30-39, 40-49, 50-59, 60-69, and 70-79 years) (Supplementary Table 1 and Fig. 1). LMS was defined as the lowest Q(Q1) of the dominant arm.

There was no difference in age among individuals in the various handgrip strength Qs $(P=0.101$ and $P=0.167$ in male and female, respectively) (Table 2). As muscle strength increased, BMI and the prevalence of obesity, hypertriglyceridemia, and metabolic syndrome increased significantly according to crude analysis $(P<$ 0.01 ) (Table 2). By contrast, the prevalence of DM decreased in both sexes $(P=0.039$ and $P=0.019$ in male and female, respectively) (Table 2).

The prevalence of obesity increased by $30 \%$ in male (odds ratio [OR], 1.300; 95\% confidence interval [CI], 1.231-1.373; $P<0.001)$ and $12 \%$ in female (OR, 1.122; 95\% CI, 1.062-1.185; $P<0.001$ ) per one $\mathrm{Q}$ of muscle strength (Supplementary Table 2). By contrast, the prevalence of DM decreased as muscle strength increased (OR per one $\mathrm{Q}, 0.926$; $95 \% \mathrm{CI}, 0.862-0.996 ; \mathrm{P}=0.039$ in male and $\mathrm{OR}$ per one $Q_{2} 0.917$; $95 \%$ CI, $0.854-0.986 ; P=0.019$ in female).

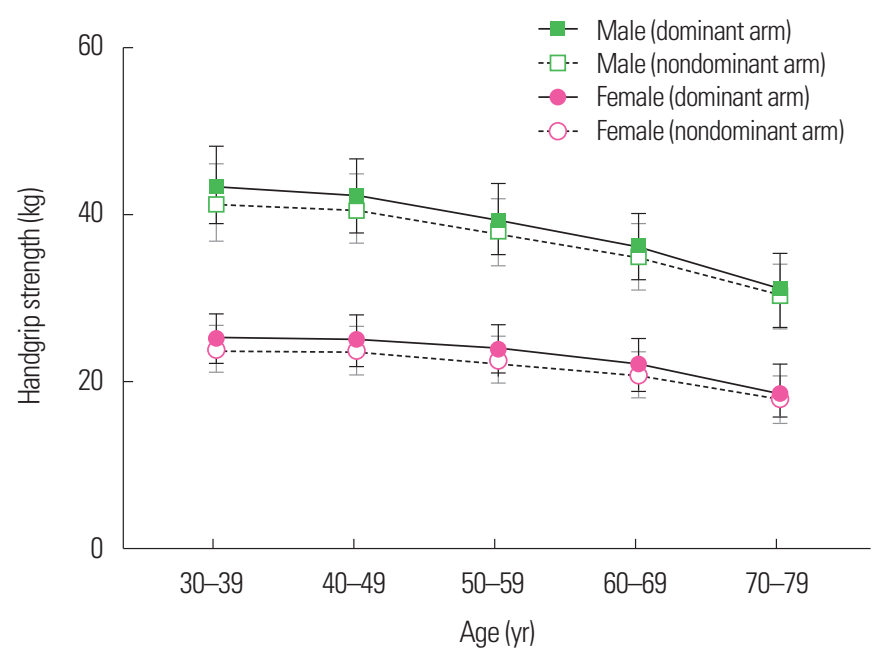

Figure 1. Handgrip strength according to age in subjects 30 to 79 years old in the 2014 to 2016 Korea National Health and Nutrition Examination Surveys. Median strength $(\mathrm{kg})$ with interquartile range in each age group is represented.

\section{The prevalence of diabetes according to LMS and obesity}

LMS was significantly associated with DM in crude analysis (OR, 1.248; 95\% CI, 1.094-1.423; $P=0.001$ ), which remained statistically significant even after adjustment for age, sex, family history of DM, abdominal obesity, dyslipidemia, and hypertension (OR, 1.328; 95\% CI, 1.133-1.558). An independent association between LMS and diabetes was found, irrespective of age (Supplementary Table 3).

As obesity was positively associated with muscle strength in both sexes (Supplementary Table 2), we subsequently analyzed the association between LMS and DM according to the presence or absence of obesity. Stratified analysis according to obesity status showed that LMS was significantly associated with diabetes in nonobese individuals (OR, 1.444; 95\% CI, 1.205-1.731; $P<$ 0.001 ) but not in those with obesity (OR, 1.200; 95\% CI, 0.982 
Table 2. Clinical characteristics according to the Qs of handgrip strength

\begin{tabular}{|c|c|c|c|c|c|}
\hline Variable & 10 & 20 & 30 & 40 & $P^{*}$ \\
\hline \multicolumn{6}{|l|}{ Male } \\
\hline Unweighted N & 1,479 & 1,459 & 1,466 & 1,456 & \\
\hline Handgrip strength, D (kg) & $31.4 \pm 0.2$ & $38.2 \pm 0.1$ & $42.3 \pm 0.1$ & $48.5 \pm 0.2$ & $<0.001$ \\
\hline Handgrip strength, ND (kg) & $31.7 \pm 0.2$ & $37.0 \pm 0.1$ & $40.3 \pm 0.1$ & $45.5 \pm 0.2$ & $<0.001$ \\
\hline Age (yr) & $50.4 \pm 0.4$ & $49.9 \pm 0.4$ & $49.8 \pm 0.4$ & $49.5 \pm 0.4$ & 0.101 \\
\hline BMI $\left(\mathrm{kg} / \mathrm{m}^{2}\right)$ & $23.8 \pm 0.1$ & $24.4 \pm 0.1$ & $24.7 \pm 0.1$ & $25.4 \pm 0.1$ & $<0.001$ \\
\hline Obesity (\%) & $32.2 \pm 1.5$ & $38.5 \pm 1.5$ & $42.3 \pm 1.6$ & $51.9 \pm 1.4$ & $<0.001$ \\
\hline Diabetes mellitus (\%) & $17.6 \pm 1.1$ & $14.1 \pm 1.1$ & $14.6 \pm 1.0$ & $14.1 \pm 1.0$ & 0.039 \\
\hline Hypertension (\%) & $30.2 \pm 1.4$ & $34.6 \pm 1.4$ & $32.7 \pm 1.5$ & $34.9 \pm 1.5$ & 0.064 \\
\hline Hypertriglyceridemia (\%) & $42.2 \pm 1.5$ & $43.6 \pm 1.6$ & $44.5 \pm 1.5$ & $46.8 \pm 1.5$ & 0.029 \\
\hline Low HDL cholesterolemia (\%) & $26.2 \pm 1.4$ & $24.4 \pm 1.4$ & $24.1 \pm 1.3$ & $24.4 \pm 1.4$ & 0.368 \\
\hline Metabolic syndrome (\%) & $35.8 \pm 1.5$ & $37.0 \pm 1.5$ & $39.5 \pm 1.5$ & $42.9 \pm 1.5$ & $<0.001$ \\
\hline \multicolumn{6}{|l|}{ Female } \\
\hline Unweighted N & 1,835 & 1,842 & 1,785 & 1,827 & \\
\hline Handgrip strength, D (kg) & $17.8 \pm 0.1$ & $22.4 \pm 0.1$ & $25.3 \pm 0.1$ & $29.5 \pm 0.1$ & $<0.001$ \\
\hline Handgrip strength, ND (kg) & $17.7 \pm 0.1$ & $21.4 \pm 0.1$ & $23.7 \pm 0.1$ & $27.3 \pm 0.1$ & $<0.001$ \\
\hline Age (yr) & $51.2 \pm 0.4$ & $50.9 \pm 0.4$ & $50.9 \pm 0.4$ & $50.5 \pm 0.4$ & 0.167 \\
\hline $\mathrm{BMI}\left(\mathrm{kg} / \mathrm{m}^{2}\right)$ & $23.3 \pm 0.1$ & $23.2 \pm 0.1$ & $23.6 \pm 0.1$ & $24.2 \pm 0.1$ & $<0.001$ \\
\hline Obesity (\%) & $29.1 \pm 1.3$ & $26.5 \pm 1.1$ & $29.8 \pm 1.3$ & $36.1 \pm 1.4$ & $<0.001$ \\
\hline Diabetes mellitus (\%) & $12.7 \pm 0.9$ & $11.8 \pm 0.8$ & $9.9 \pm 0.8$ & $10.5 \pm 0.8$ & 0.019 \\
\hline Hypertension (\%) & $23.8 \pm 1.2$ & $23.7 \pm 1.1$ & $25.8 \pm 1.2$ & $25.5 \pm 1.1$ & 0.155 \\
\hline Hypertriglyceridemia (\%) & $21.7 \pm 1.3$ & $22.2 \pm 1.1$ & $22.2 \pm 1.2$ & $23.4 \pm 1.2$ & $<0.001$ \\
\hline Low HDL cholesterolemia (\%) & $40.8 \pm 1.4$ & $38.6 \pm 1.4$ & $36.1 \pm 1.2$ & $43.4 \pm 1.3$ & 0.326 \\
\hline Metabolic syndrome (\%) & $27.3 \pm 1.3$ & $27.3 \pm 1.2$ & $26.7 \pm 1.2$ & $32.5 \pm 1.3$ & 0.007 \\
\hline
\end{tabular}

Values are presented as mean or prevalence \pm standard error.

*Logistic and linear regression analysis without any adjustment.

$\mathrm{Q}$, quartile; $\mathrm{D}$, dominant arm; ND, nondominant arm; BMI, body mass index; $H D L$, high-density lipoprotein.

Table 3. OR for diabetes mellitus in the stratified analysis according to the presence or absence of obesity

\begin{tabular}{|c|c|c|c|c|}
\hline \multirow{2}{*}{ Variable } & \multicolumn{2}{|c|}{ Unadjusted } & \multicolumn{2}{|c|}{ Multivariable regression analysis* } \\
\hline & $\mathrm{OR}(95 \% \mathrm{Cl})$ & $P$ & $\mathrm{OR}(95 \% \mathrm{Cl})$ & $P$ \\
\hline \multicolumn{5}{|l|}{ Nonobese } \\
\hline Low muscle strength & $1.444(1.205-1.731)$ & $<0.001$ & $1.513(1.224-1.870)$ & $<0.001$ \\
\hline Family history of diabetes & $2.355(1.993-2.783)$ & $<0.001$ & $3.532(2.838-4.394)$ & $<0.001$ \\
\hline Abdominal obesity & $3.046(2.491-3.724)$ & $<0.001$ & $1.386(1.039-1.849)$ & 0.027 \\
\hline Hypertriglyceridemia & $2.852(2.443-3.329)$ & $<0.001$ & $2.088(1.683-2.591)$ & $<0.001$ \\
\hline Low HDL cholesterolemia & $2.175(1.863-2.539)$ & $<0.001$ & $1.565(1.261-1.943)$ & $<0.001$ \\
\hline Hypertension & $3.919(3.378-4.546)$ & $<0.001$ & $1.610(1.318-1.967)$ & $<0.001$ \\
\hline Age & $1.078(1.072-1.085)$ & $<0.001$ & $1.079(1.070-1.089)$ & $<0.001$ \\
\hline Sex (female) & $0.578(0.500-0.669)$ & $<0.001$ & $0.556(0.454-0.681)$ & $<0.001$ \\
\hline \multicolumn{5}{|l|}{ Obese } \\
\hline Low muscle strength & $1.200(0.982-1.468)$ & 0.075 & $1.124(0.879-1.437)$ & 0.351 \\
\hline Family history of diabetes & $2.180(1.812-2.622)$ & $<0.001$ & $2.653(2.142-3.287)$ & $<0.001$ \\
\hline Abdominal obesity & $2.165(1.770-2.649)$ & $<0.001$ & $1.751(1.371-2.236)$ & $<0.001$ \\
\hline Hypertriglyceridemia & $1.453(1.240-1.701)$ & $<0.001$ & $1.547(1.244-1.922)$ & $<0.001$ \\
\hline Low HDL cholesterolemia & $1.657(1.387-1.979)$ & $<0.001$ & $1.476(1.176-1.854)$ & 0.001 \\
\hline Hypertension & 2.369 (2.006-2.798) & $<0.001$ & 1.621 (1.315-1.999) & $<0.001$ \\
\hline Age & $1.048(1.042-1.055)$ & $<0.001$ & 1.051 (1.042-1.059) & $<0.001$ \\
\hline Sex (female) & $0.930(0.793-1.090)$ & 0.370 & $0.795(0.651-0.970)$ & 0.024 \\
\hline
\end{tabular}

*Age, sex, muscle strength, family history of diabetes, abdominal obesity, hypertriglyceridemia, low HDL cholesterolemia, and hypertension were included in the multivariable regression analysis.

$\mathrm{OR}$, odds ratio; $\mathrm{Cl}$, confidence interval; $\mathrm{HDL}$, high-density lipoprotein. 
$1.468 ; P=0.075$ ) (Table 3). Adjustment for age, sex, family history of DM, abdominal obesity, dyslipidemia, and hypertension also confirmed a significant association between diabetes and LMS only in those without obesity (OR, 1.513; 95\% CI, 1.224-1.870; $P<0.001$ and $\mathrm{OR}, 1.124 ; 95 \% \mathrm{CI}, 0.879-1.437 ; P=0.351$ in nonobese and obese participants, respectively) (Table 3). Further BMI-stratified analyses in nonobese individuals confirmed that an independent association between LMS and diabetes was only found in those with a BMI of less than $23 \mathrm{~kg} / \mathrm{m}^{2}$ (Supplementary Table 4).

In the obese population, age $(P<0.001)$, male sex $(P=0.024)$, family history of diabetes (OR, 2.653; 95\% CI, 2.142-3.287), abdominal obesity (OR, 1.751; 95\% CI, 1.371-2.236), hypertriglyceridemia (OR, 1.547; 95\% CI, 1.244-1.922), low HDL cholesterolemia (OR, 1.476; 95\% CI, 1.176-1.854), and hypertension (OR, $1.621 ; 95 \% \mathrm{CI}, 1.315-1.999)$ were significantly associated with the presence of diabetes in multivariable analysis (Table 3).

\section{DISCUSSION}

In the current study, muscle strength was significantly associated with BMI irrespective of sex. Although high muscle strength positively correlated with the prevalence of obesity, it was significantly associated with a low risk of DM even after making adjustments for risk factors. Obesity-stratified analysis revealed that the protective effect of muscle strength for DM was found only in those without obesity. There was no significant association between muscle strength and DM in the obese population.

Currently, muscle strength for the definition of sarcopenia is based on the absolute muscle strength: the suggested cutoffs for handgrip strength are less than $26 \mathrm{~kg}$ for males, and less than $16 \mathrm{~kg}$ for females per the Foundation for the National Institutes of Health Biomarkers Consortium Sarcopenia Project', or less than $27 \mathrm{~kg}$ for males and less than $16 \mathrm{~kg}$ for females per the European Working Group on Sarcopenia in Older People ${ }^{8}$, respectively. However, there may exist ethnic differences in the reference ranges for muscle strength. ${ }^{22}$ In addition, in the case of middle-aged individuals, muscle strength can affect metabolic health and mortality even if above the range indicating sarcopenia ${ }^{23}$; therefore, age-specific definitions of LMS should be considered for the prediction of health outcomes among age-matched populations. We defined age-specific
LMS using a nationwide database representative of the Korean population, and found that it had an independent association with $\mathrm{DM}$ in the nonobese group.

A previous study using KNHANES data applied a unifying definition of LMS considering the entire population irrespective of age and also found a significant association between LMS and diabetes. ${ }^{7}$ The authors ${ }^{7}$ used weight-normalized muscle strength; however, the relationship between body mass and muscle strength might be different between obese and nonobese individuals. ${ }^{15}$ Furthermore, one's handgrip ability is relatively independent of weightbearing movement, and no difference of absolute handgrip power between obese and nonobese subjects was reported. ${ }^{15}$

Interestingly, in the current study, the association between muscle strength and diabetes was significant only in the nonobese population. Obesity is a well-known and strong risk factor for developing diabetes ${ }^{24}$, and it was positively correlated with muscle strength in the current study, which might attenuate the effect of muscle strength on diabetes. Obesity was defined according to BMI in the current study, and BMI is based on body weight. As BMI cannot differentiate between individuals with large muscle mass or large fat mass ${ }^{25}$, some number of those individuals with obesity may in fact have large muscle mass, which attenuates the effect of muscle strength on diabetes in those with obesity in the current study. However, further stratified analysis according to BMI in those without obesity confirmed that an independent association between LMS and diabetes was only found in those with a BMI of less than $23 \mathrm{~kg} / \mathrm{m}^{2}$, which also suggests LMS might be more important in those with low BMI.

The current study has several limitations. The first one is its crosssectional nature. We could not determine the causality in the association between LMS and the risk of diabetes because of the combined cross-sectional study design. As skeletal muscle is the primary tissue responsible for insulin-mediated glucose disposal, low skeletal muscle mass reduces insulin-mediated glucose disposal, independent of obesity. The independent association between skeletal muscle and insulin resistance has been confirmed by previous epidemiological ${ }^{26,27}$ and experimental ${ }^{28}$ studies. The second limitation is that we had no data on muscle mass. There were no data available on body composition from the 2014 to 2016 KNHANESs. Muscle mass is another important determinant of sarcopenia 
and has been reported to increase the risk of $\mathrm{DM}^{2}$, cardiovascular diseases ${ }^{3}$, and long-term mortality. ${ }^{4}$ However, a previous study confirmed that muscle strength is more important than muscle mass for future clinical outcomes ${ }^{5}$, and we successfully showed that an independent association exists between diabetes and LMS in a nonobese population.

In conclusion, LMS was independently associated with DM in a Korean population aged between 30 and 79 years old. Obesitystratified analysis revealed that it was significant only in the nonobese population, which suggests that, even in the nonobese population, LMS might be a risk factor for developing diabetes. Resistance training can be an efficient tool for the prevention of diabetes in the population with normal body weight.

\section{CONFLICTS OF INTEREST}

The author declares no conflict of interest.

\section{SUPPLEMENTARY MATERIALS}

Supplementary Table 1. Handgrip strength $(\mathrm{kg})$ in each age group.

Supplementary Table 2. Behavior of the risk of obesity, diabetes mellitus, and metabolic syndrome as the $\mathrm{Q}$ of handgrip strength increased.

Supplementary Table 3. The association between low muscle strength and diabetes according to age.

Supplementary Table 4. OR for diabetes mellitus in the stratified analysis according to $\mathrm{BMI}$ in those without obesity.

They can be found via https://doi.org/10.7570/jomes.2019.28. 1.46.

\section{REFERENCES}

1. Larsen BA, Wassel CL, Kritchevsky SB, Strotmeyer ES, Criqui $\mathrm{MH}$, Kanaya AM, et al. Association of muscle mass, area, and strength with incident diabetes in older adults: the Health $\mathrm{ABC}$ study. J Clin Endocrinol Metab 2016;101:1847-55.

2. Jang HC. Sarcopenia, frailty, and diabetes in older adults. Diabetes Metab J 2016;40:182-9.
3. Harada K, Suzuki S, Ishii H, Aoki T, Hirayama K, Shibata Y, et al. Impact of skeletal muscle mass on long-term adverse cardiovascular outcomes in patients with chronic kidney disease. Am J Cardiol 2017;119:1275-80.

4. Kim JH, Lim S, Choi SH, Kim KM, Yoon JW, Kim KW, et al. Sarcopenia: an independent predictor of mortality in community-dwelling older Korean men. J Gerontol A Biol Sci Med Sci 2014;69:1244-52.

5. Newman AB, Kupelian V, Visser M, Simonsick EM, Goodpaster BH, Kritchevsky SB, et al. Strength, but not muscle mass, is associated with mortality in the health, aging and body composition study cohort. J Gerontol A Biol Sci Med Sci 2006;61: $72-7$.

6. Jurca R, Lamonte MJ, Barlow CE, Kampert JB, Church TS, Blair SN. Association of muscular strength with incidence of metabolic syndrome in men. Med Sci Sports Exerc 2005;37: 1849-55.

7. Lee MR, Jung SM, Bang H, Kim HS, Kim YB. Association between muscle strength and type 2 diabetes mellitus in adults in Korea: Data from the Korea national health and nutrition examination survey (KNHANES) VI. Medicine (Baltimore) 2018;97:e10984.

8. Cruz-Jentoft AJ, Bahat G, Bauer J, Boirie $Y$, Bruyère $\mathrm{O}$, Cederholm T, et al. Sarcopenia: revised European consensus on definition and diagnosis. Age Ageing 2019;48:16-31.

9. Studenski SA, Peters KW, Alley DE, Cawthon PM, McLean RR, Harris TB, et al. The FNIH sarcopenia project: rationale, study description, conference recommendations, and final estimates. J Gerontol A Biol Sci Med Sci 2014;69:547-58.

10. Gale CR, Martyn CN, Cooper C, Sayer AA. Grip strength, body composition, and mortality. Int J Epidemiol 2007;36: 228-35.

11.Lee MR, Jung SM, Kim HS, Kim YB. Association of muscle strength with cardiovascular risk in Korean adults: findings from the Korea National Health and Nutrition Examination Survey (KNHANES) VI to VII (2014-2016). Medicine (Baltimore) 2018;97:e13240.

12. Hardy R, Cooper R, Aihie Sayer A, Ben-Shlomo Y, Cooper C, Deary IJ, et al. Body mass index, muscle strength and physical performance in older adults from eight cohort studies: the 
HALCyon programme. PLoS One 2013;8:e56483.

13. Rantanen T, Harris T, Leveille SG, Visser M, Foley D, Masaki $\mathrm{K}$, et al. Muscle strength and body mass index as long-term predictors of mortality in initially healthy men. J Gerontol A Biol Sci Med Sci 2000;55:M168-73.

14. Hasan NA, Kamal HM, Hussein ZA. Relation between body mass index percentile and muscle strength and endurance. Egypt J Med Hum Genet 2016;17:367-72.

15. Hulens M, Vansant G, Lysens R, Claessens AL, Muls E, Brumagne S. Study of differences in peripheral muscle strength of lean versus obese women: an allometric approach. Int J Obes Relat Metab Disord 2001;25:676-81.

16. Choi YJ, Kim HC, Kim HM, Park SW, Kim J, Kim DJ. Prevalence and management of diabetes in Korean adults: Korea National Health and Nutrition Examination Surveys 1998-2005. Diabetes Care 2009;32:2016-20.

17. Pfeffermann D. The use of sampling weights for survey data analysis. Stat Methods Med Res 1996;5:239-61.

18. Koo BK, Kim SW, Yi KH, Moon MK. Low economic status is identified as an emerging risk factor for diabetes mellitus in Korean men aged 30 to 59 years in Korean National Health and Nutrition Examination Survey 2008 to 2010. Diabetes Metab J 2015;39:137-46.

19. Grundy SM, Cleeman JI, Daniels SR, Donato KA, Eckel RH, Franklin BA, et al. Diagnosis and management of the metabolic syndrome: an American Heart Association/National Heart, Lung, and Blood Institute Scientific Statement. Circulation 2005;112:2735-52.

20. Lee SY, Park HS, Kim DJ, Han JH, Kim SM, Cho GJ, et al. Appropriate waist circumference cutoff points for central obe- sity in Korean adults. Diabetes Res Clin Pract 2007;75:72-80.

21. World Health Organization Western Pacific Region. The AsiaPacific perspective: redefining obesity and its treatment. Sydney: Health Communications Australia; 2000.

22. Dodds RM, Syddall HE, Cooper R, Kuh D, Cooper C, Sayer AA. Global variation in grip strength: a systematic review and meta-analysis of normative data. Age Ageing 2016;45:209-16.

23. Sasaki H, Kasagi F, Yamada M, Fujita S. Grip strength predicts cause-specific mortality in middle-aged and elderly persons. Am J Med 2007;120:337-42.

24. Field AE, Coakley EH, Must A, Spadano JL, Laird N, Dietz $\mathrm{WH}$, et al. Impact of overweight on the risk of developing common chronic diseases during a 10-year period. Arch Intern Med 2001;161:1581-6.

25. Frankenfield DC, Rowe WA, Cooney RN, Smith JS, Becker D. Limits of body mass index to detect obesity and predict body composition. Nutrition 2001;17:26-30.

26. Lim S, Kim JH, Yoon JW, Kang SM, Choi SH, Park YJ, et al. Sarcopenic obesity: prevalence and association with metabolic syndrome in the Korean Longitudinal Study on Health and Aging (KLoSHA). Diabetes Care 2010;33:1652-4.

27.Lee SW, Youm Y, Lee WJ, Choi W, Chu SH, Park YR, et al. Appendicular skeletal muscle mass and insulin resistance in an elderly Korean population: the Korean social life, health and aging project-health examination cohort. Diabetes Metab J 2015;39:37-45.

28. Skov-Jensen C, Skovbro M, Flint A, Helge JW, Dela F. Contraction-mediated glucose uptake is increased in men with impaired glucose tolerance. Appl Physiol Nutr Metab 2007;32: 115-24. 